\title{
Classification of Web Pages by Using Particle Swarm Optimization Algorithm
}

\section{Muhammad Hamid Abdulraheem}

ghaydabdulaziz@uomosul.edu.iq

Computer and Internet Center

\section{Received on: 29/06/2011}

\section{University of Mosul, Mosul, Iraq}

Ghayda A.A. Al-Talib

College of Computer Sciences and Mathematics

Accepted on: 03/10/2011

\section{ABSTRACT}

As the amount of information available on the internet grows so does the need for more effective data analysis methods. This paper utilizes the particle swarm optimization (PSO) algorithm in the field of web content classification, and used part of speech tagging algorithm to reduce the large numbers of attributes associated with web content mining. The proposed algorithm gave a good classification accuracy, which comparable to the accuracy of Ant-miner algorithm and acquire less training time.

Keywords: web content classification, PSO,

$$
\begin{aligned}
& \text { تصنيف صفحات الثبكة العنكبوتية باستخدام خوارزمية أمثَكَة عناصر السرب } \\
& \text { غيداء عبد العزيز الطالب عمد حامد عبد الرحيم }
\end{aligned}
$$

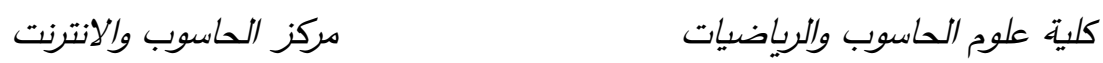

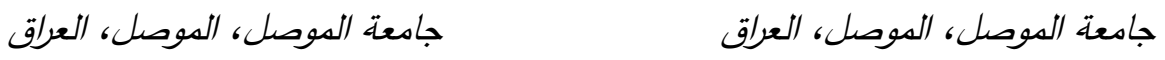

$$
\begin{aligned}
& \text { تاريخ قبول البحث: 2011/10/03 } \\
& \text { تاريخ استلام البحث: 2011/06/29 }
\end{aligned}
$$

مع نمو المعلومات المتوفرة على الشبكة العالمية Internet ازدادت الحاجة إلى طرائق أكثر فاعلية في

تحليل البيانات، في هذا البحث استخدمت خوارزمية أمثَكة عناصر السرب (PSO) بأسلوب جديد في تصنيف المحتوى النصي لصفحات الثبكة العنكبوتية مع استخدام خوارزمية وسم أجزاء الكلام بوصفها وسيلة لتقليل السمات، واظهر أسلوب الخوارزمية المقترح دقة تصنيف جيدة ومقاربة لدقة تصنيف خوارزمية النمل في التتقيب

$$
\text { Ant-Miner }
$$

الكلمات المفتاحية: تصنيف المحتوى النصي لصفحات الثبكة العنكبوتية، خوارزمية أمثَلَة عناصر السرب

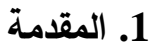

أصبحت المعلومات هي سمة العصر الذي نعيشه حتى أن البعض أطلق عليه عصر المعلومات،

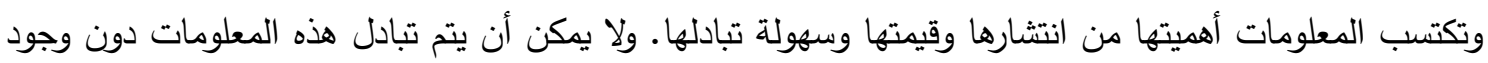

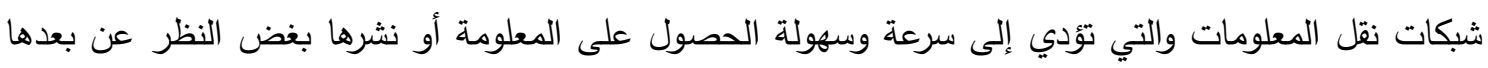

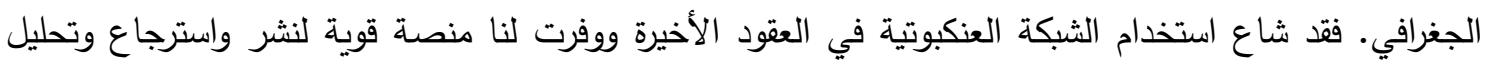

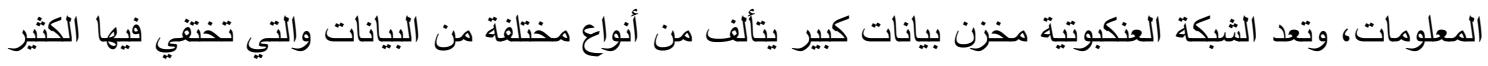

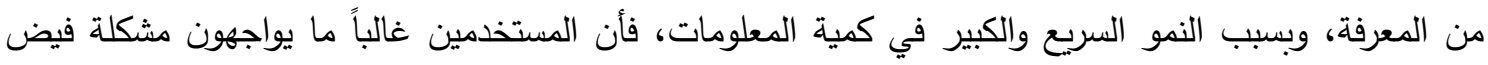

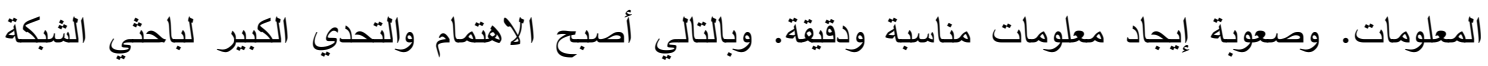
العنكبوتية هو تثخيص مسألة كفاءة وفعالية أنظمة إدارة واسترجاع المعلومات المبنية على الثبكة العنكبوتية [18].

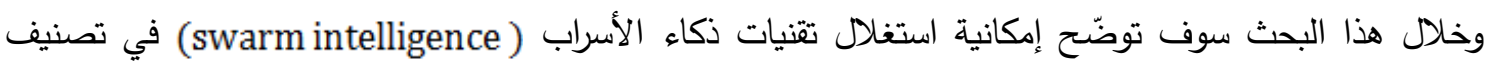


عام 2004 استخدم الباحثان (Holden and Freitas) [8]، خوارزمية (Ant-Miner) في مجال تصنيف محتوى الثبكة العنكبوتية، واظهر البحث أن خوارزمية (Ant-Miner) كانت أكثر فعالية من خوارزمية التصنيف (C5.0)، وأيضا تم التحقق من جدوى بعض تقنيات المعالجة المبنية على اللغة لتقليل عدد السمات.

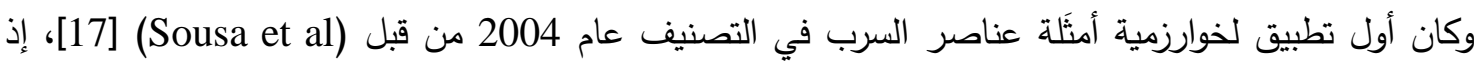
اقترحوا استخدام خوارزمية أمتَكة عناصر الأسراب بوصفها أداة جديدة للتتقيب في البيانات ومثلوا ثلاثة نسخ لخوارزمية أمثَلة عناصر السرب وهم: خوارزمية أمثلة عناصر الأسراب المتقطعة (discreet pso)، وخوارزمية أمتَّة عناصر السرب ذات الانحدار الخطي للأوزان (linear decreasing weight pso) وخوارزمية أمثلَّة عناصر السرب المحددة (Constricted pso). وقورنت نتائجها مع الخوارزمية الجينية وخوارزمية شجرة الاستقراء، وتبين أن خوارزمية أمثَكة عناصر السرب كانت أداة مرشحة ومنافسة لمهام التصنيف.

3. التنقيب في الثبكة العنكبوتية (Web Mining)

يهدف التتقيب في الثبكة العنكبوتية إلى اكتثاف المعرفة من مصادر بيانات ضخمة متوفرة على الثبكة

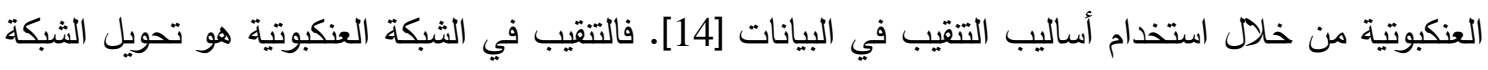
العنكبوتية إلى بيئة أكثر فائدة ويمكن لمستخدمها إيجاد المعلومات التي يحتاجها بسهولة وسرعة، وتتضمن كذلك الك التيات اكتشاف وتحليل البيانات، والوثائق والوسائط المتعددة في الثبكة العنكبوتية [16]. ويمكن تصنيف التتقيب في بيانات الشبكة العنكبوتية إلى ثلاثة أنواع بناءً على أهداف التتقيب والذي يحدد التخصص الذي سوني سوف يُنقب فئب فيه من الثبكة العنكبوتية:

1 التنقيب في محتوى الثبكة العنكبوتية Web content mining

2. التتقيب في هيكل الثبكة العنكبوتية التبكية

3eb usage mining التنقيب في استخدام الثبكة العنكبوتية فيخدية التتقيب في محتوى الثبكة العنكبوتية يحاول اكتثاف معلومات قيّمة من محتويات الثبكة العنكبوتية، وبصورة عامة محتوى الثبكة العنكبوتية يشير إلى المحتويات النصية وأيضا بوصفه مصطلحاً بديلاً يطلق عليه التتقيب في النص (text mining)، ومن ابرز مهام التتقيب في المحتوى هما التصنيف والعنقدة، أما التتقيب في لهي هيكل الشبكة العنكبوتية فأنه يضم نمذجة مواقع الثبكة العنكبوتية من حيث هياكل الارتباطات التشعبية. إن معلومات الربط المتبادل التي يتم الحصول عليها يمكن أن تستخدم لإيجاد صفحات بناءً على التثابه أو الصلة

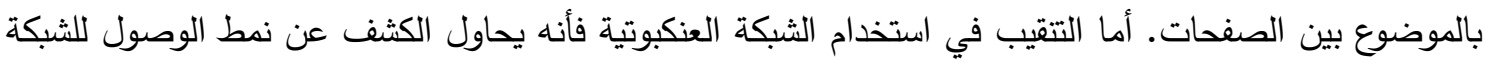
العنكبوتية من خلال بيانات جلسة المستخدم (user data session) المسجلة في ملف أداء الشبكة العنكبوتية .[18]،[11] (Web log file)

4. أهمية وتطبيقات تصنيف صفحات الشبكة العنكبوتية [14]

تصنيف صفحات الثبكة العنكبوتية، هي عملية تخصيص الصفحة إلى واحد أو أكثر من الأصناف المعرفة مسبقاً، والتصنيف غالبا ما يطرح بوصفه مسألة تعليم بالأشراف والذي فيه مجموعة من بيانات محددة

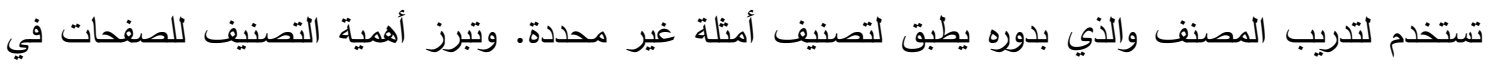

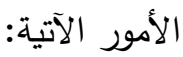


1. إنثاء وإدامة وتوسيع أدلة البحث على الثبكة العنكبوتية.

2. تحسين نوعية نتائج البحث: إذ إن الغموض في الاستعلام هو من بين المشاكل التي تقوض نتائج عملية البحث.

3. الأنظمة المساعدة لإجابة الأسئلة (Helping question answering systems): في هذه الأنظمة ربما

تستخدم تقنية التصنيف لتحسين جودة الجواب.

4. الباحث الموجه (focused crawling): إن الباحث الموجه حول موضوع محدد يهتم بالوثائق التي لها علاقة

بمجموعة من المواضيع المحددة مسبقا.

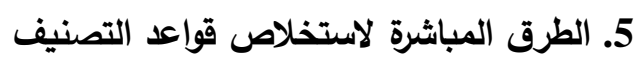

تستخدم غالباً خوارزمية التغطية المتسلسلة (sequential covering algorithm) لاستخلاص قواعد

تصنيف من البيانات مباشرة. تتمو القواعد بطريقة مفرطة (greedy) وفق مقياس تقييم محدد. فتستخلص خوارزمية

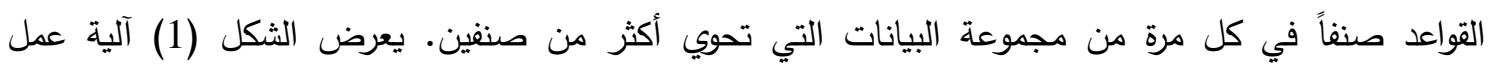
خوارزمية التغطية المتسلسلة لمجموعة البيانات التي تتضمن مجموعة من الأمثلة الإيجابية والسلبية. تبدأ خوارزمية لئية

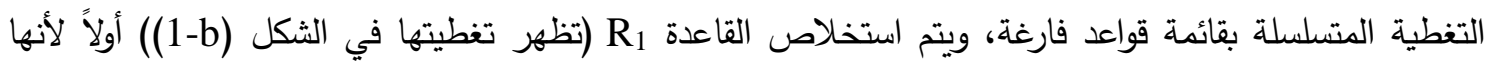

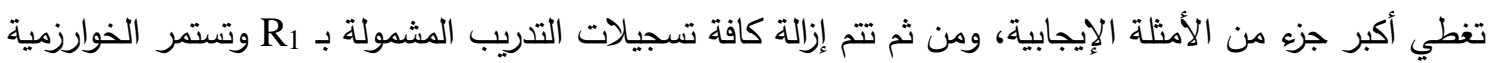
في البحث عن أفضل قاعدة تالية، وهي R2. تكون القاعدة مقبولة إذا كانت تغطي معظم الأمثلة الإيجابية ولا تغطي أياً من أو القليل جداً من الأمثلة السلبية، وبمجرد إيجاد قاعدة كهذه تتم إزالة تسجيلات التدريب المشمولة

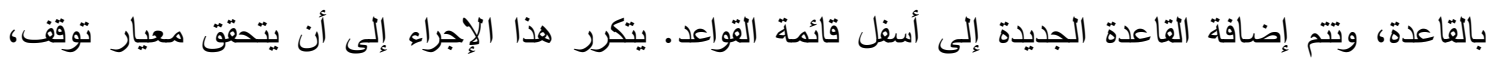

تستمر الخوارزمية بعدها لتوليد قواعد من أجل الصنف التالي [1]ة.

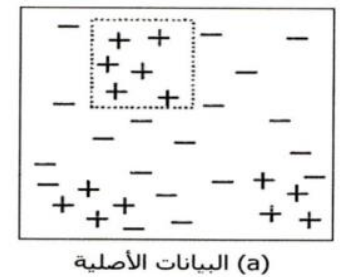

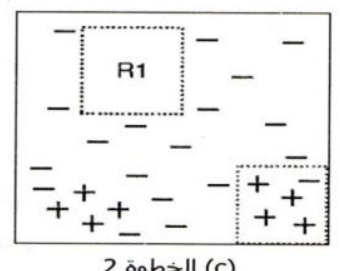
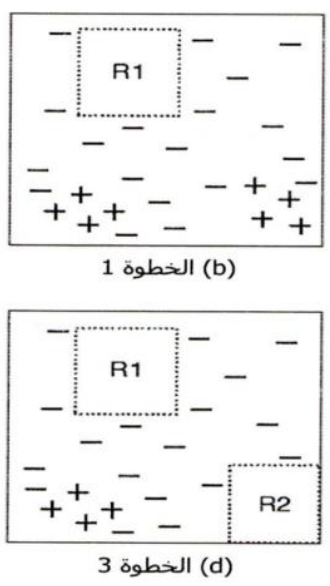

الثكل (1). مثال عن خوارزمية التغطية المتسلسلة [1].

6. استخدام المعالجة اللغوية في تقليل واختيار السمات

1-6] Part-of-speech-Tagging

يعرف وسم أجزاء الكلام من خلال تخصيص وسم أو ملحق مفرد لكل كلمة في نص اللغة الانكليزية حسب الصنف اللغوي الذي تتمي إليه الكلمة، وفي اللغة الانكليزية تصنف الكلمات إلى: الأسماء، الأفعال، 


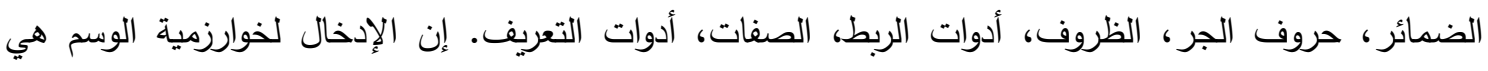

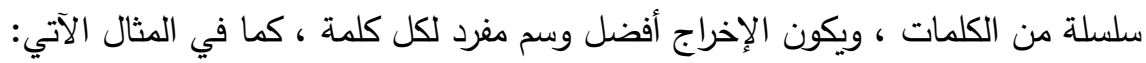
The/DT guys/NNS that/WDT make/VBP traditional/JJ hardware/NN are/VBP really/RB being/VBG obsolete/VBN by/IN microprocessor-based/JJ machines/NNS ,/, "/" said/VBD Mr./NNP Benton/NNP ./.

[7] ( stemming (إرجاع الكلمات إلى جذورها

وهي عملية اختزال صيغ مختلفة للكلمة إلى جذر مشترك، وخوارزمية التجذير هي الخوارزمية التي تزيل

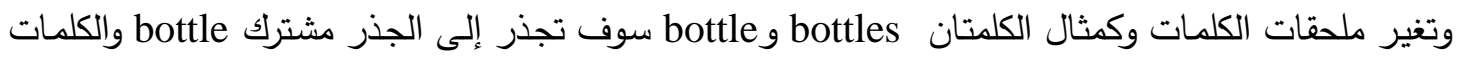

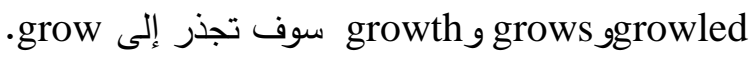

7. استخدام معامل كسب المعلومات (information gain) في تقليل السمات [4] في مسائل التصنيف يحسب معامل كسب المعلومات لسمات مسألة التصنيف بالاعتماد على معامل التباين (Entropy)، بوصفه مرحلة مسبقة لغرض تقليل تلك السمات. ويعرف معامل التباين بأنه مقياس نظري

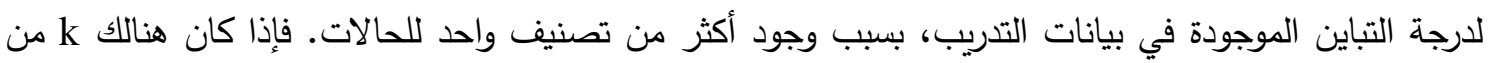

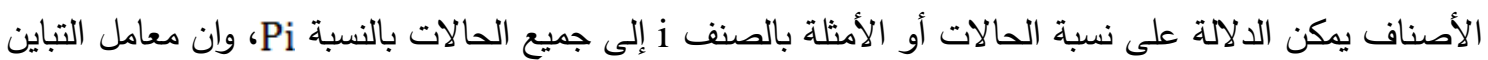
$\mathrm{E}=-\sum_{\mathrm{i}=1}^{\mathrm{k}} \mathrm{Pi} \log _{2}$

لبيانات التدريب يرمز له E ويحسب من خلال المعادلة (1):

$$
\begin{aligned}
& \text { إذ أن: i i تمثل الصنف وتتغير من } 1 \text { إلى k من الأصناف. }
\end{aligned}
$$

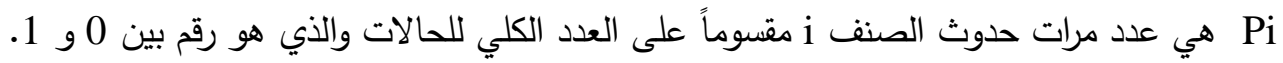

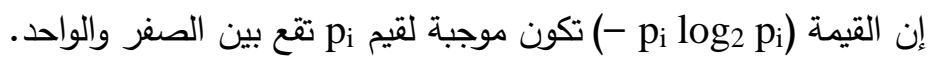

8 8 هوارزمية أمثَّة عناصر السرب Particle Swarm Optimization

$$
\text { يقوم سرب الطيور على المبادئ الأساسية الآتية [3]: }
$$

1. التجانس Homogeneity: كل طائر في السرب له أنموذج التصرف نفسه، فالسرب يتحرك بدون قائد. 2. المحلية Locality: حركة كل طائر فقط تتأثر بحركة السرب الأقرب للطائر . 3. تجنب التصادم Collision Avoidance: البقاء بالقرب من رفاق السرب مع عدم التصادم.

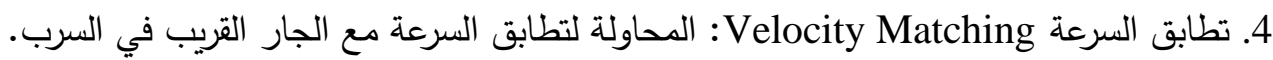
5. انضمام السرب Flock Centering: محاولة البقاء بقرب الرفاق المجاورين في السرب.

$$
\text { إن خوارزمية أمثَكة عناصر السرب طورت بناء على النموذج الآتي: [15] }
$$

1. عندما يحدد احد الطيور في السرب الهدف أو الطعام (أو اكبر قيمة لدالة الهدف) فان الطائر حالا ينقل

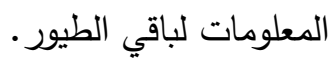

2. باقي الطيور تتجذب إلى الهدف أو الطعام تدريجيا. 3. هنالك إمكانيات لكل طائر منها التفكير الذاتي و تذكر أفضل موقع سابق. 
إذاً فان هذا النموذج يحاكي عملية بحث عشوائي في فضاء بحث للوصول إلى أفضل قيمة لدالة الهدف، وبتكرار ذلك فان الطيور ستصل تدريجيا للهدف.

9. خوارزمية أمثَّة عناصر السرب المتقطعة (19،5،6] (DPSO: The discrete PSO Algorithm) إن خوارزمية (DPSO) تعالج المتغيرات المتقطعة (السمات) مباشرة، وتختلف عن خوارزمية (PSO)

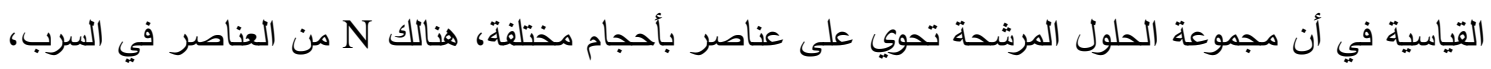
وحجم كل عنصر يتغير من 1 إلى n؛ إذ إن n هو عدد السمات في المسألة، وفي هذا السياق فأن حجم العنصر

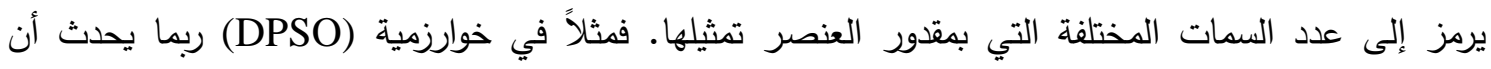

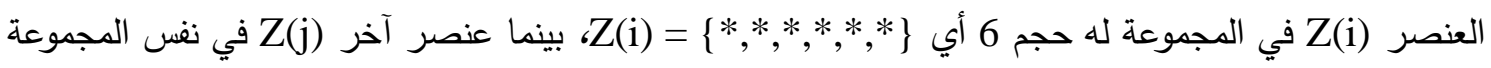
له الحجم \} Z(i) =\{*, أي 2، وهكذا لباقي العناصر • وكل عنصر Z(i) يحتفظ بسجل لأفضل موقع زاره على الإطلاق، وتلك المعلومة تخزن في متجه آخر يسمى B(i)، كذلك يحتفظ السرب بسجل لأفضل موقع عمومي

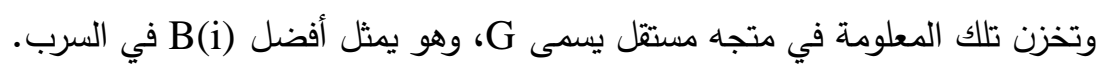

1-9 ترميز العنصر في خوارزمية (DPSO)

تمثل كل سمة بفهرس، وتتغير تلك الأرقام من 1 إلى n، فالعنصر عبارة عن مجموعة فرعية من الفهارس غير مرتبة وبدون تكرار فمثلا العنصر k قد يمثل بالفهارس وكما يأتي: $\mathrm{Z}(\mathrm{k})=\{2,4,18,1\}, \quad \mathrm{k} \in\{1,2, \ldots, \mathrm{N}\}$.

2-9 توليد المجتمع الأولي في خوارزمية (DPSO)

لإنشاء المجتمع الأولي الذي تبدأ به الخوارزمية، فأن N من المتجهات بحجم n تولد عشوائياً، وفي

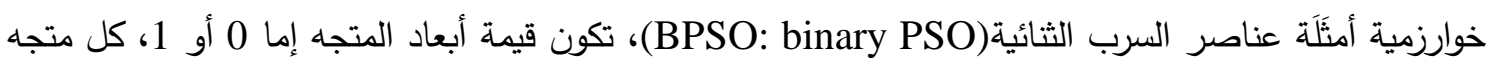
يولد بصورة مستقلة، وكل بعد في المتجه Y(i) يحسب من خلال سحب رقم عشوائي $\boldsymbol{~ ف ي ~ ا ل ف ت ر ة ~ ( 0 , 1 ) ، ~}$ فإذا كان Y(i,d)= إن طريقة توليد المجتمع الأولي في خوارزمية (DPSO) مطابقة لطريقة توليد المجتمع الأولي في خوارزمية وكما يلي: (BPSO) إن فهرس كل سمة لها قيمة 1 تتسخ إلى الحل الجديد أو العنصر، فمثلا إذا كان العنصر الأولي المولد

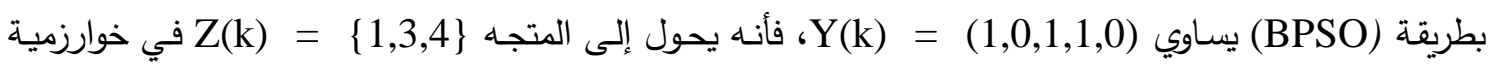
كتهيئة أولية للعناصر ، بسبب أن قيم السمات A1,A3,A4 في المتجه تساوي 1. إن ابتداء العناصر

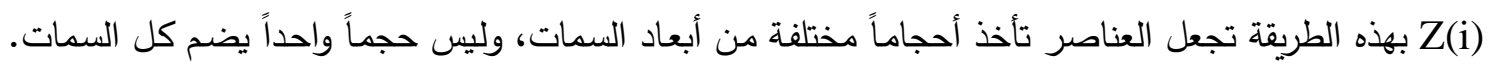

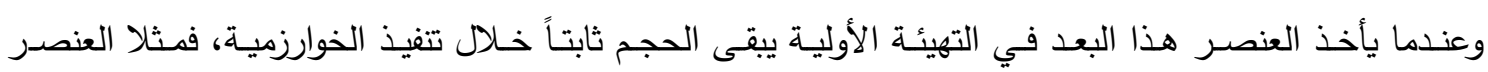
الذي ابتدأ بأربعة فهارس للسمات سوف يحمل دائماً أربعة فهارس، ومن المحتمل أن تتغير أرقام الفهارس في كل وقت يحدِّث العنصر موقعه. 
إن خوارزمية (DPSO) لا تستخدم متجه السرعة كما في خوارزمية (PSO) القياسية، فهي تتعامل مع

الاحتمالية النسبية. إن خوارزمية (DPSO) تستخدم المصفوفة M(i) لتمثيل الاحتمالية النسبية التي تقابل السمات

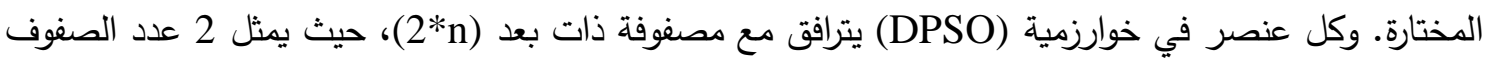
و nدد الأعمدة. حيث إن عدد الأعمدة يعادل عدد سمات المسألة وتمثل قيم الصف الأول والثاني كما يأتي: $M(i)=\left\{\begin{array}{l}\text { proportiord }- \text { likelihood }- \text { row } \\ \text { attribute }- \text { index }- \text { row }\end{array}\right\}$

كل بعد في الصف الأول لهذه المصفوفة يمثل احتمالية اختيار السمة التي تقابلها في الصف الثاني، وهذا يعني وجود علاقة واحد إلى واحد بين الصف الأول والصف الثاني للمصفوفة، بدايةً فأن جميع القيم في الصف الأول للمصفوفة M(i) تأخذ قيمة 1، فمثلا إذا كان لدينا خمس سمات تكون المصفوفة M(i) كما يأتي: $M(i)=\left(\begin{array}{ccccc}1.0 & 1.0 & 1.0 & 1.0 & 1.0 \\ 1 & 2 & 3 & 4 & 5\end{array}\right)$

وبعد التوليد الأولي للعناصر، تُحدّث تلك المصفوفة دائماً قبل اشتقاق العنصر الجديد المرتبط مع تلك المصفوفة.

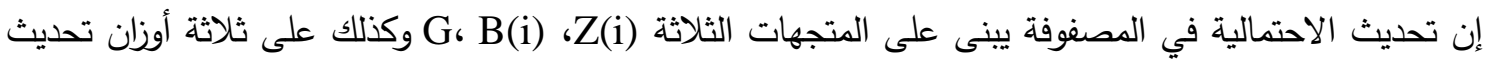

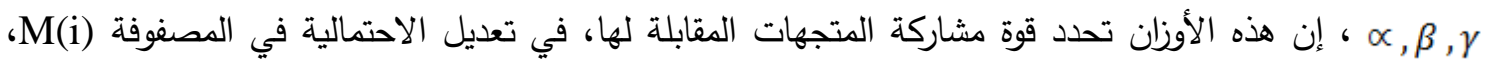

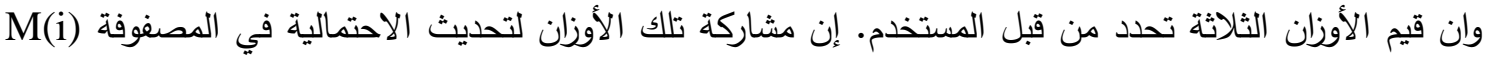
تتم كما يأتي:

جميع الفهارس الموجودة في المتجه Z(i) تزيد الاحتمالية النسبية للفهارس المقابلة لها في المصفوفة اللفهارس بمقدار ه. إضافة إلى ذلك فأن جميع الفهارس الموجودة في المتجه B(i) تزيد الاحتمالية النسبية في المصفوفة M(i) للفهارس التي تقابلها في المصفوفة بمقدار م ، والثيء نفسه بالنسبة للمتجه G الذي لئيد الاحتمالية النسبية في المصفوفة M(i) للعناصر التي يحتويها G بمقدار ر • فمثلا: Z(i) = لو كانت والمصفوفة (i) $M(i)=\left(\begin{array}{ccccc}1.0 & 1.0 & 1.0 & 1.0 & 1.0 \\ 1 & 2 & 3 & 4 & 5\end{array}\right)$

فان تحديث الاحتمالية في المصفوفة يكون كالتالي: $M(i)=\left(\begin{array}{ccccc}(1.0) & (1.0+\alpha+\beta+\gamma) & (1.0+\alpha+\beta) & (1.0+\alpha) & (1.0+\beta+\gamma) \\ 1 & 2 & 3 & 4 & 5\end{array}\right)$

إن المصفوفة الناتجة الجديدة M(i) تحل محل المصفوفة القديمة، وتُستخدم لتوليد عنصر جديد، وهو ما يقابل تحديث الموقع في خوارزمية (PSO) القياسية. 4-9 تحديث موقع العناصر في خوارزمية (DPSO): إن المصفوفة M(i) تستخدم لتحديث أو توليد حالة جديدة من العنصر Z(i) المرتبط مع المصفوفة M(i) فهنالك سلسلة من العمليات تتجز على مصفوفة الاحتمالية، ففي البداية يضرب كل رقم احتمالية في الصف 
الأول في المصفوفة برقم عشوائي بين(0,1)، حيث يسحب رقم عشوائي جديد لكل رقم في الصف الأول. ولتوضيح ذلك نفترض أن المصفوفة : $M(i)=\left(\begin{array}{ccccc}1.00 & 1.36 & 1.22 & 1.10 & 1.26 \\ 1 & 2 & 3 & 4 & 5\end{array}\right)$

عندها يضرب الصف الأول بالأرقام العشوائية وكما يلي: $M(i)=\left(\begin{array}{ccccc}(1.00 \times \varphi 1) & (1.36 \times \varphi 2) & (1.22 \times \varphi 3) & (1.10 \times \varphi 4) & (1.26 \times \varphi 5) \\ 1 & 2 & 3 & 4 & 5\end{array}\right)$

إذ إن (ب5, , , , ب1) هي أرقام عشوائية منتظمة مسحوبة بصورة مستقلة في الفترة (0,1) وعلى افتراض أن $M(i)=\left(\begin{array}{ccccc}0.11 & 0.86 & 0.57 & 0.62 & 1.09 \\ 1 & 2 & 3 & 4 & 5\end{array}\right)$ المصفوفة الناتجة تكون:

وأن الموقع الجديد لقيم الفهارس المرتبطة مع قيم الاحتمالية يعرف من خلال ترتيب الاحتمالية النسبية في الصف $M(i)=\left(\begin{array}{ccccc}1.09 & 0.86 & 0.62 & 0.57 & 0.11 \\ 5 & 2 & 4 & 3 & 1\end{array}\right)$

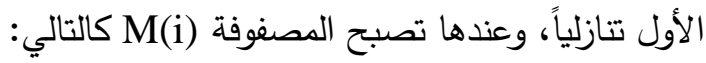

العملية التالية على المصفوفة هي اختيار الفهارس من الصف الثاني التي ستثكل موقع العنصر الجديد، بعد ترتيب المصفوفة يختار عدد Si من الفهارس من الصف الثاني من اليسار إلى اليمين لتمثل تحديث موقع العنصر

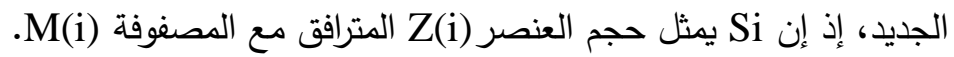

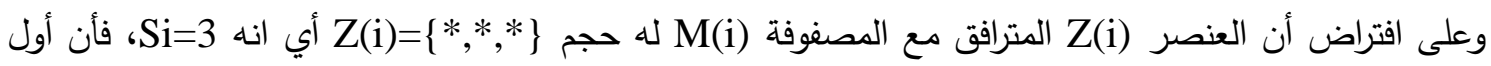
ثلاثة فهارس من الصف الثاني سوف تختار لتثكيل العنصر الجديد ويصبح: $\}$ Z(i) = الفهارس التي لها احتمالية نسبية أعلى هي أكثر احتمالية لان تختار، وهكذا لكل عناصر السرب. 10. التحويرات والإضافات إلى خوارزمية (DPSO) مع أمثَكَة تطبيقية ونتائج من البحث 1-10 طريقة الابتداء للعناصر الأولية للسرب إن جميع خوارزميات أمثلة عناصر السرب المطبقة تبتدئ المجتمع الأولي لها من خلال قيم عشوائية يأخذها عناصر السرب، أي أن القاعدة الواحدة التي تمثل عنصراً من عناصر السرب تولد أولاً عشوائياً، وهذا قد

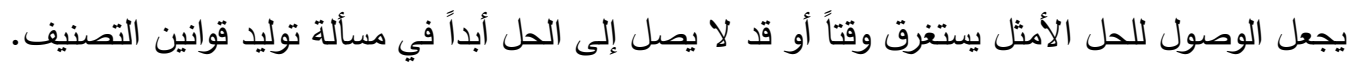

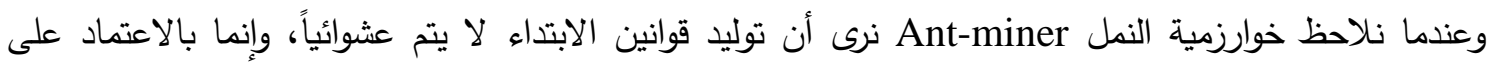

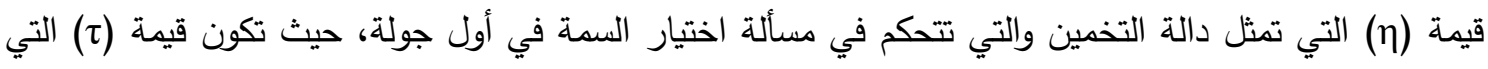
تمثل إفراز النمل في البداية متساوية لجميع المسالك، ثم مع مرور الزمن تتحكم قيمة (ح) بالسيطرة على اختيار

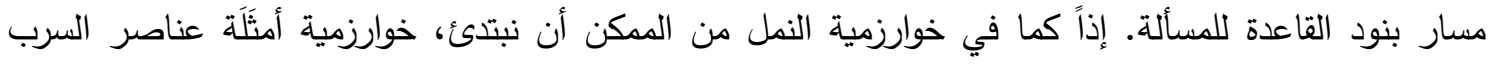
المتقطعة بالاعتماد على قيمة دالة تخمين في اختيار تجمعات القواعد المولدة ابتداءً.

2-10 دالة التخمين المضافة إلى الخوارزمية (DPSO)

دالة التخمين التي استخدمت في هذا البحث تُحسب لكل بند (سمة=قيمة) في مسألة التصنيف، فهي تعبر عن أهمية البند للمسألة، ولإغراض التتقيب في البيانات فأن تلك الأهمية للسمة تقاس عادة بعدد الحالات التي 
غُطّّت بواسطة قيمة سمة معينة مع صنف ثابت، لذلك يمكن تعريف قيمة دالة التخمين لأهمية البند في المسألة $\eta_{i, k}=\frac{\mid V_{i}=\text { Value }_{k} \text { \& class } C \mid}{\mid V_{i}=\text { Value }_{k} \mid}$

كما في المعادلة رقم (1): (1) - (1)

إذ إن: وهذا يعني انه كل بند في بيانات التدريب يأخذ عدد من قيم لـ $\eta$ بقدر عدد الأصناف في المسألة. يتم حساب دالة التخمين مسبقاً لكل بند، وبقدر عدد الأصناف في المسألة كما موضح في فئ المثال لئل الآتي:

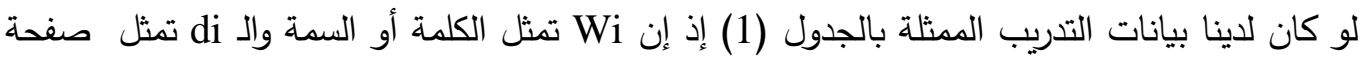

شبكة. وقيم السمات في الجدول هي إما ورود الكلمة (1) أو عدم ورودها (0). جدول (1). مصفوفة التدريب

\begin{tabular}{|c|c|c|c|c|c|c|c|}
\hline & W1 & W2 & W3 & W4 & W5 & W6 & الصنف| \\
\hline d1 & 1 & 1 & 0 & 0 & 0 & 0 & a \\
\hline d2 & 0 & 1 & 1 & 1 & 0 & 0 & b \\
\hline d3 & 1 & 1 & 0 & 0 & 0 & 1 & a \\
\hline d4 & 1 & 0 & 1 & 0 & 1 & 1 & c \\
\hline d5 & 0 & 0 & 0 & 0 & 1 & 1 & c \\
\hline d6 & 0 & 0 & 1 & 1 & 0 & 0 & b \\
\hline
\end{tabular}

ويمكن تمثيل فضاء أو مخطط البحث لهذا المثال كما في الثكل (2).

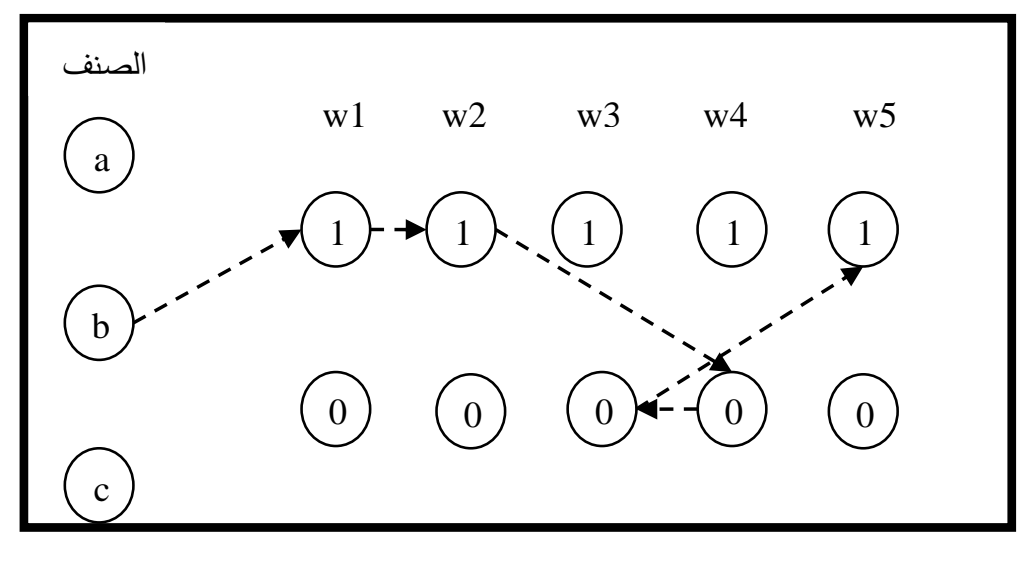

الثكل (2). فضاء (مخطط) البحث

وفي هذا المخطط الدائري المتجه، أي رأس في المخطط ممكن أن يرتبط مع أي رأس آخر وفي مسارات مخطط لا يتكرر المرور على نفس السمة أكثر من مرّة في المسار الواحد. ولحساب قيمة دالة التخمين ض لرؤوس المخطط في

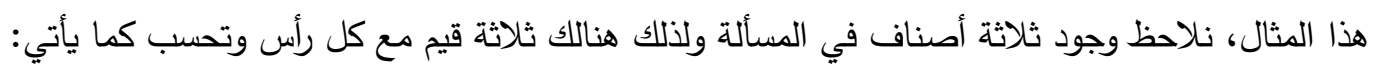
للرأس (W1=1) تحسب قيمة ך مع الصنف a كما يأتي: إذ إن السمة W1 بقيمة تساوي 1 وردت مع الصنف a مرتين، وان السمة w1 بقيمة 1 تكرر ثلاث مرات بغض

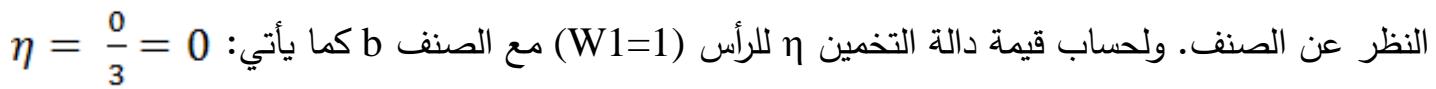


إذ إن السمة W1 بقيمة 1 لم ترد مع الصنف b، وان السمة W1 بالقيمة 1 تكررت ثلاث مرات.

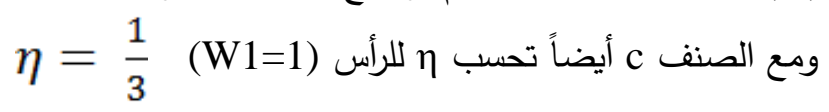

إذ إن السمة W1 بالقيمة 1 وردت مع الصنف c مرة واحدة، وان السمة W1 بالقيمة 1 تكررت ثلاث مرات.

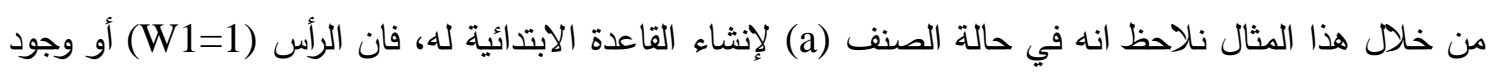

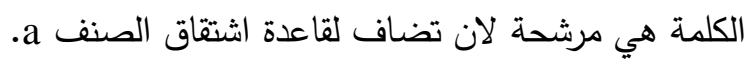
والجدول (2) يمثل قيم ض لكل الرؤوس ولكل الأصناف في المثال السابق لثلاث سمات مع قيمتها. الجدول (1). يمثل قيم دالة التخمين لبنود المسألة

\begin{tabular}{|r|r|r|r|r|r|r|}
\hline السمة & $\mathrm{W} 1=1$ & $\mathrm{~W} 1=0$ & $\mathrm{~W} 2=1$ & $\mathrm{~W} 2=0$ & $\mathrm{~W} 3=1$ & $\mathrm{~W} 3=0$ \\
\hline $\mathrm{a}$ & $2 / 3$ & 0 & $2 / 3$ & 0 & 0 & $2 / 3$ \\
\hline $\mathrm{b}$ & 0 & $2 / 3$ & $1 / 3$ & $1 / 3$ & $2 / 3$ & 0 \\
\hline $\mathrm{c}$ & $1 / 3$ & $1 / 3$ & 0 & $2 / 3$ & $1 / 3$ & $1 / 3$ \\
\hline $\mathrm{d}$ & $\mathrm{W} 4=1$ & $\mathrm{~W} 4=0$ & $\mathrm{~W} 5=1$ & $\mathrm{~W} 5=0$ & $\mathrm{~W} 6=1$ & $\mathrm{~W} 6=0$ \\
\hline $\mathrm{a}$ & 0 & $2 / 4$ & 0 & $2 / 4$ & $1 / 3$ & $1 / 3$ \\
\hline $\mathrm{a}$ & 1 & 0 & 0 & $2 / 4$ & 0 & $2 / 3$ \\
\hline $\mathrm{b}$ & 0 & $2 / 4$ & 1 & 0 & $2 / 3$ & 0 \\
\hline $\mathrm{c}$ & & & & & & \\
\hline
\end{tabular}

عندما تبدأ الخوارزمية بإعطاء القيم الأولية لعناصر السرب، تبتدئ الخوارزمية بصنف معين مثلاً (a) ويتم أيجاد

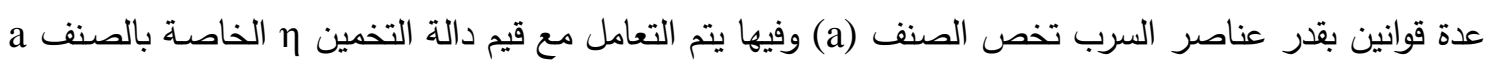

بعد حساب قيمة دالة التخمين $\eta$ لكل رأس في فضاء البحث للمسألة ، يتم حساب احتمالية اختيار كل تجمع أو رأس (Pi,k) وحسب المعادلة (2): $P_{i, k}=\frac{\eta_{i k}}{\sum_{i=1}^{a} X_{i}^{*} \sum_{k=1}^{b i} \eta_{i k k}}$

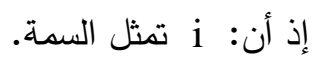
k تمثل قيمة دالة التخمين للتجمع. تأخذ قيمة 1 إذا كانت السمة i لم تستخدم بعد في القاعدة وتأخذ قيمة 0 عدا ذلك. a

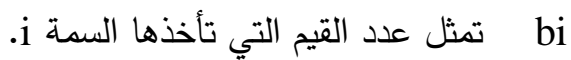
إن هذه المعادلة تكرر عند اختيار كل سمة يراد إضافتها للقاعدة، ولغرض تطبيق القاعدة على المثال السابق لإيجاد قاعدة تصنيف للصنف (a)، تحسب الاحتمالية بالاعتماد على قيم (ך) للصنف (a) فقط وحسب الجدول 
جدول (3). قيم دالة التخمين والاحتمالية لبنود الدسألة مع الصنف

\begin{tabular}{|c|c|c|c|c|c|c|}
\hline التجمع & $\mathrm{W} 1=1$ & $\mathrm{~W} 1=0$ & $\mathrm{~W} 2=1$ & $\mathrm{~W} 2=0$ & $\mathrm{~W} 3=1$ & $\mathrm{~W} 3=0$ \\
\hline a قيم ךلصنف a & $2 / 3$ & 0 & $2 / 3$ & 0 & 0 & $2 / 3$ \\
\hline $\mathrm{P}_{\mathrm{i}, \mathrm{k}}$ & 0.182 & 0 & 0.182 & 0 & 0 & 0.182 \\
\hline التجدع & $\mathrm{W} 4=1$ & $\mathrm{~W} 4=0$ & $\mathrm{~W} 5=1$ & $\mathrm{~W} 5=0$ & $\mathrm{~W} 6=1$ & $\mathrm{~W} 6=0$ \\
\hline a قيم ךلصنف a & 0 & $2 / 4$ & 0 & $2 / 4$ & $1 / 3$ & 1.3 \\
\hline $\mathrm{P}_{\mathrm{i}, \mathrm{k}}$ & 0 & 0.136 & 0 & 0.136 & 0.1 & 0.1 \\
\hline
\end{tabular}

فمثلاً للتجمع (W1=1) يتم حساب Pi,k كما يأتي وبالاعتماد على المعادلة (2):

$P_{w 1=1}=\frac{\frac{2}{3}}{\frac{2}{3}+\frac{2}{3}+\frac{2}{3}+\frac{2}{4}+\frac{2}{4}+\frac{1}{3}+\frac{1}{3}}=(0.182)$

وبعد حساب الاحتمالية لكل التجمعات يتم تطبيق طريقة عجلة الروليت (Roulette wheel) لاختيار

إحدى التجمعات لأضافتها للقاعدة، وبعد اختيار تجمع معين، تعاد حسابات الاحتمالية من جديد لاستثناء السمة

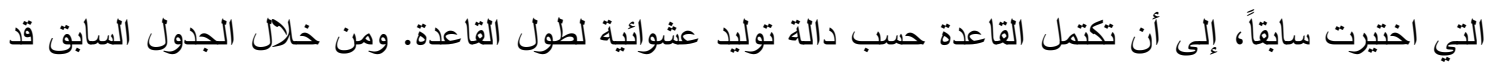
يولد القاعدة الآتية: - n

If $\mathrm{W} 2=1$ and $\mathrm{W} 4=0$ and $\mathrm{W} 1=1$ then $\mathrm{a}$

إن هذه القاعدة تمثل احد عناصر السرب، وبعد توليد جميع عناصر السرب بهذه الطريقة يكون قد تكون لدينا سرب من الطيور يبحث عن القاعدة الأمثل لصنف معين، وان هذا السرب قد أخذ قيم أولية غير عشوائية.

4-10 تحوير في طريقة اختيار بنود القاعدة في خوارزمية (DPSO)

فمن خلال ملاحظة خوارزمية (DPSO) في المصادر [19, 6, 5] نجد أن الخوارزمية (DPSO) طُبِقت لاختيار سمات وليس تجمع (سمة =قيمة)، وهذا الاختيار للسمة يأتي كعملية مسبقة لخوارزميات التصنيف التقليدية.

إذاً يمكن توسعة هذا الأسلوب لخوارزمية (DPSO) عن طريق الأمور الآتية:

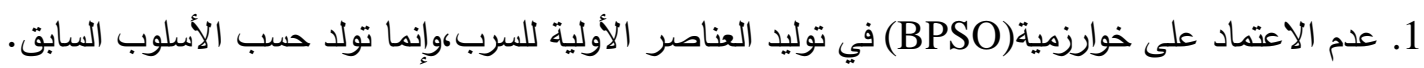
2. ترميز العنصر: في خوارزمية (BPSO) كل عنصر يمثل بمتجه من الأرقام الصحيحة التي تمثل فهرس توليد

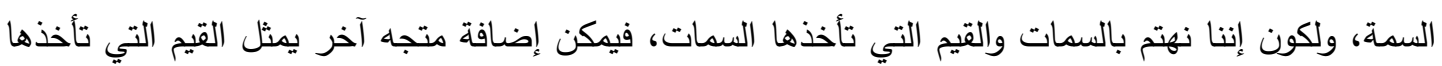
فهرس تلك السمة، فمثلاً المتجه (i) يكون للفهارس والمتجه الآخر (ik) لقيم تلك الفهارس:

$\mathrm{Z}(\mathrm{i})=\{2,4,18,1\}$

$\mathrm{Z}(\mathrm{ik})=\{0,1,1,0\}$

هذا هو التمثيل البرمجي لعنصر السرب، وهو يكافئ التمثيل المنطقي للقاعدة الآتية: If $\mathrm{W} 2=0$ and $\mathrm{W} 4=1$ and $\mathrm{W} 18=1$ and $\mathrm{W} 1=0$ Then a

وبعد توليد جميع العناصر في السرب، يقيم كل عنصر بواسطة دالة الجودة المستخدمة في مسائل التقيب في

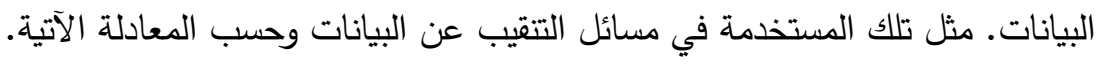


$Q=\frac{T P}{T P+F N} * \frac{T N}{F P+T N}$

إذ إن (true positive) TP عدد الحالات التي غطتها القاعدة في جزء الشرط والتي لها نفس الصنف في ناتج القاعدة.

FP (false positive) في ناتج القاعدة.

FN (false negative) القاعدة. TN (true negative) القاعدة. ويعد العنصر الذي له أفضل قيمة لدالة الجودة هو أفضل عنصر في السرب ويرمز له G، وأيضاً كل عنصر

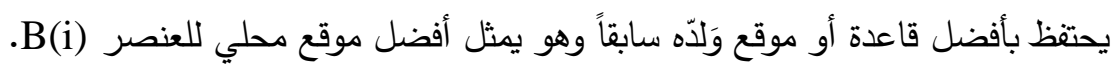
5-10 تمثيل السرعة بواسطة الاحتمالية النسبية: كما مَرّ سابقاً في خوارزمية DPSO فان كل عنصر يأتي معه مصفوفة M(i بأبعاد (2*n) حيث أن

$$
\text { هي عدد السمات في المسألة. }
$$

ولكوننا نهتم بالسمة وبالقيمة التي تأخذها السمة إذاً تكون المصفوفة بأبعاد (2n٪2) بسبب أن كل سمة ممكن أن

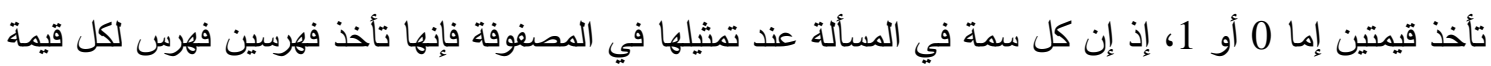
فمثلاً السمة رقم 2 في العنصر Z(i) في المثال السابق تمثل في المصفوفة M(i) من خلال الفهرسين الجديدين

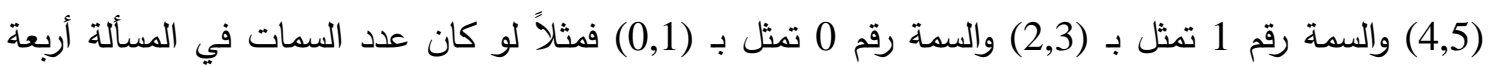

$M(i)=\left\{\begin{array}{cccccccc}1.0 & 1.0 & 1.0 & 1.0 & 1.0 & 1.0 & 1.0 & 1.0 \\ 0 & 1 & 2 & 3 & 4 & 5 & 6 & 7\end{array}\right\}$

تكون المصفوفة M(i) كالآتي:

حيث يمثل الصف الأول الاحتمالية النسبية كما مرّ سابقاً، أيضاً تحدث المصفوفة M(i) التالية لكل عنصر من خلال قيم تصاعدياً، والقيم التي تختار من المصفوفة لتمثل العنصر الجديد ترجع إلى قيم الفهارس الأصلية، فمثلاً إذا كانت المصفوفة M(i) بعد جمع قيم الاحتمالية النسبية بالمعاملات $M(i)=\left\{\begin{array}{cccccccc}1.40 & 1.30 & 1.2 & 0.9 & 0.8 & 0.7 & 0.6 & 0.5 \\ 6 & 3 & 5 & 1 & 4 & 7 & 2 & 0\end{array}\right\}$

وعلى افتراض أن طول العنصر السابق كان 3. إذاً يعاد توليد عنصر جديد بنفس الطول ولكن بفهارس جديدة، ففي

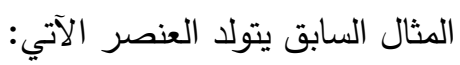

$\mathrm{Z}(\mathrm{i})=\{3,1,2\}$

$\mathrm{Z}(\mathrm{ik})=\{1,0,0\}$

إن توليد هذا العنصر يتم من خلال. قسمة الفهرس المأخوذة من المصفوفة M(i) على الرقم 2 ويؤخذ الجزء الصحيح من الناتج ويوضع في متجه الفهرس، أما متجه القيمة المقابل لمتجه الفهرس فتحسب فيما إذا كان متبقي

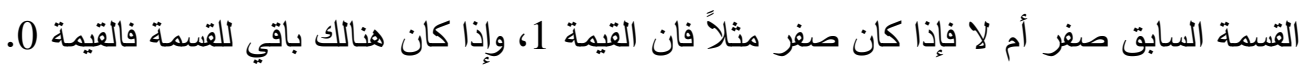




\section{6-10 ملخص الخوارزمية المقترحة}

يمكن تلخيص الخوارزمية (dpso) المقترحة كما في المخطط الانسيابي في الثكل (3)، والتي تكتثف قاعدة تصنيف واحدة في كل تتفيذ، وحسب الخطوات الآتية:

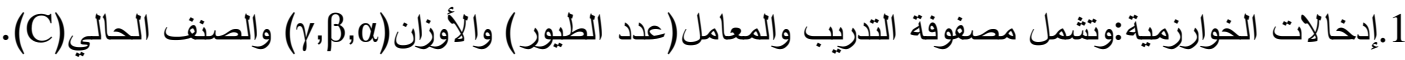

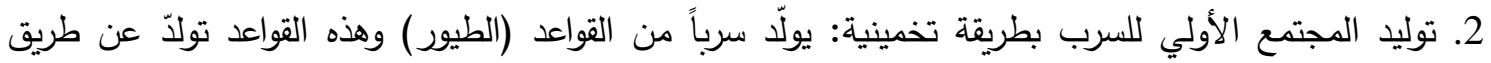
الاحتمالية لقيم الدالة التخمينية خ وحسب الصنف الحالي، وليست عشوائيا، ولضمان بساطة وشمولية القواعد

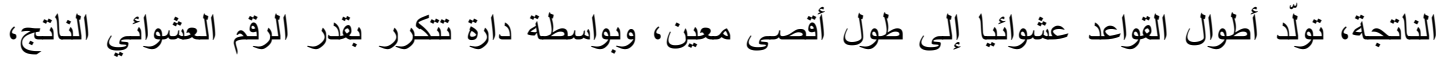

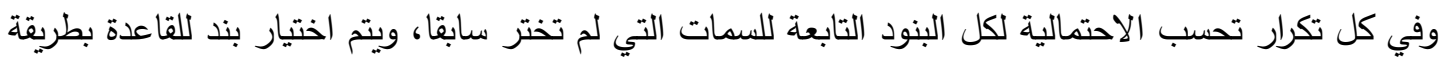
عجلة الروليت، تكرر العملية السابقة بقدر عدد طيور السرب N، وتحسب جودة كل قاعدة وتخزن القواعد في لئي

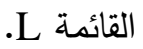

3. اختيار أفضل موقع محلي لكل طائر : يحتفظ كل طائر بأفضل موقع مرّ به، وبداية يأخذ هذا المتغير (RL) قيمة القاعدة الحالية (R). (R).

4. اختيار أفضل قاعدة في عامّة السرب(RG):بالاعتماد على أعلى قيمة لجودة القواعد المخزنة في القائمة(L). 5. اعتماد مبدأ الطيور في البحث: ولكل قاعدة في القائمة L تحسب لها السرعة الجديدة ويحدّث موقعها أي توليد قاعدة جديدة، ويحدّث أفضل موقع محلي لكل طائر، إذ يتم تبديل أفضل موقع محلي بالموقع الجديد في حال كان أفضل جودة منه(RL=R).

6. توليد مجتمعات جديدة للسرب: بعد تحريك السرب في الخطوة السابقة (5) تكون القواعد قد تغيرت، إذ يتم حساب أفضل قاعدة في السرب من جديد (RG*) بالاعتماد على قيم دالة الجودة، وتقارن مع أفضل قاعدة من

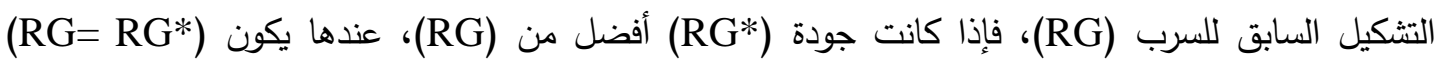
وكذلك الجودة (QRG=QRG*)، بحيث يحتفظ المتغير (RG) دائماً بأفضل قاعدة من بين القواعد العمومية لكل مجتمعات السرب، وفي حال لم تكن جودة (RG*) أفضل من (RG)، يتم فقط تحديث عداد التقارب ويستمر التكرار في دارة توليد مجتمعات السرب لجولة جديدة من تجديد السرعة وحساب الموقع الجديد لقواعد

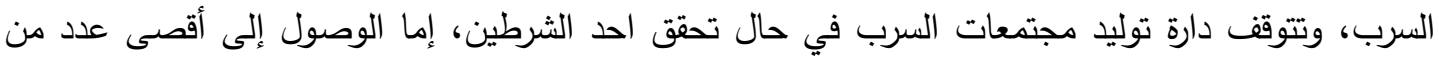

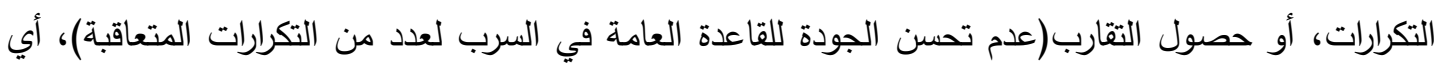
عندما يصل عداد التقارب إلى عدد محدد مسبقا للتقارب، وبعد الخروج من الدارة تُنتِج الخوارزمية أفضل قاعدة

$$
\text { للصنف الحالي. }
$$

\section{7-10 خوارزمية التغطية المتعاقبة المغلفة لخوارزمية (DPSO)}

يمكن استغلال خوارزمية التغطية المتعاقبة كما في المخطط الانسيابي للخوارزمية في الثكل (4)، لكي تكون الإطار الخارجي لخوارزمية (DPSO)، وفيها يمكن إيجاد قاعدة تصنيف واحدة في الزمن الواحد. حيث تبدأ خوارزمية التغطية من خلال تهيئة مجموعة القوانين (RS: rule set) بمجموعة فارغة، ثم لكل صنف تتجز الخوارزمية عدد من تكرارات الإيعاز (WHILE) وان كل تكرار ينفذ خوارزمية (DPSO) مرّة واحدة، والتي ترجّع

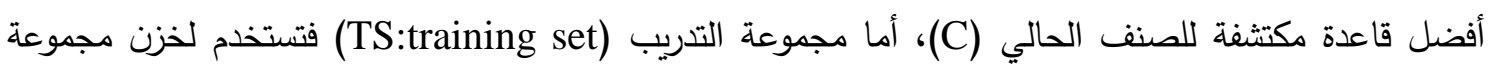
بيانات التدريب والتي سوف تثتق منها قواعد التصنيف، بعد ذلك أفضل قاعدة مكتشفة تضاف إلى مجموعة 
القواعد، بعد أن تمّ تثذيبها لإزالة البنود التي لأتثارك في تحسين جودة القاعدة، وأن الحالات التي غطتها القاعدة

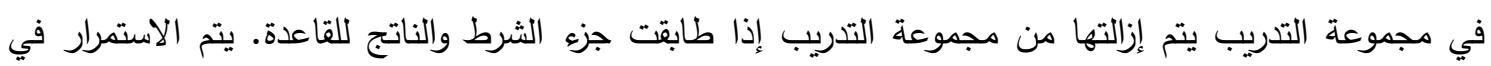
التكرار (WHILE) ما دام عدد الحالات الغير مغطاة لذلك الصنف المحدد (C) هو اكبر من حد عتبة معين

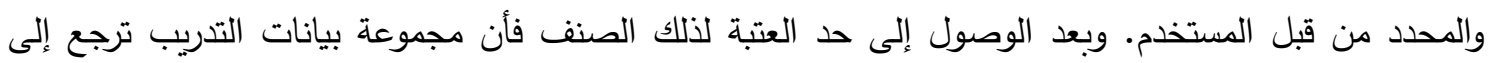
حالتها الأولية التي تضم جميع حالات التدريب وتبدأ الخوارزمية بإيجاد قوانين التصنيف للصنف التالي، وبعد التيد اكتمال جميع الأصناف في المسألة، ترتب قائمة القوانين المكتشفة حسب جودة القاعدة تتازليا.

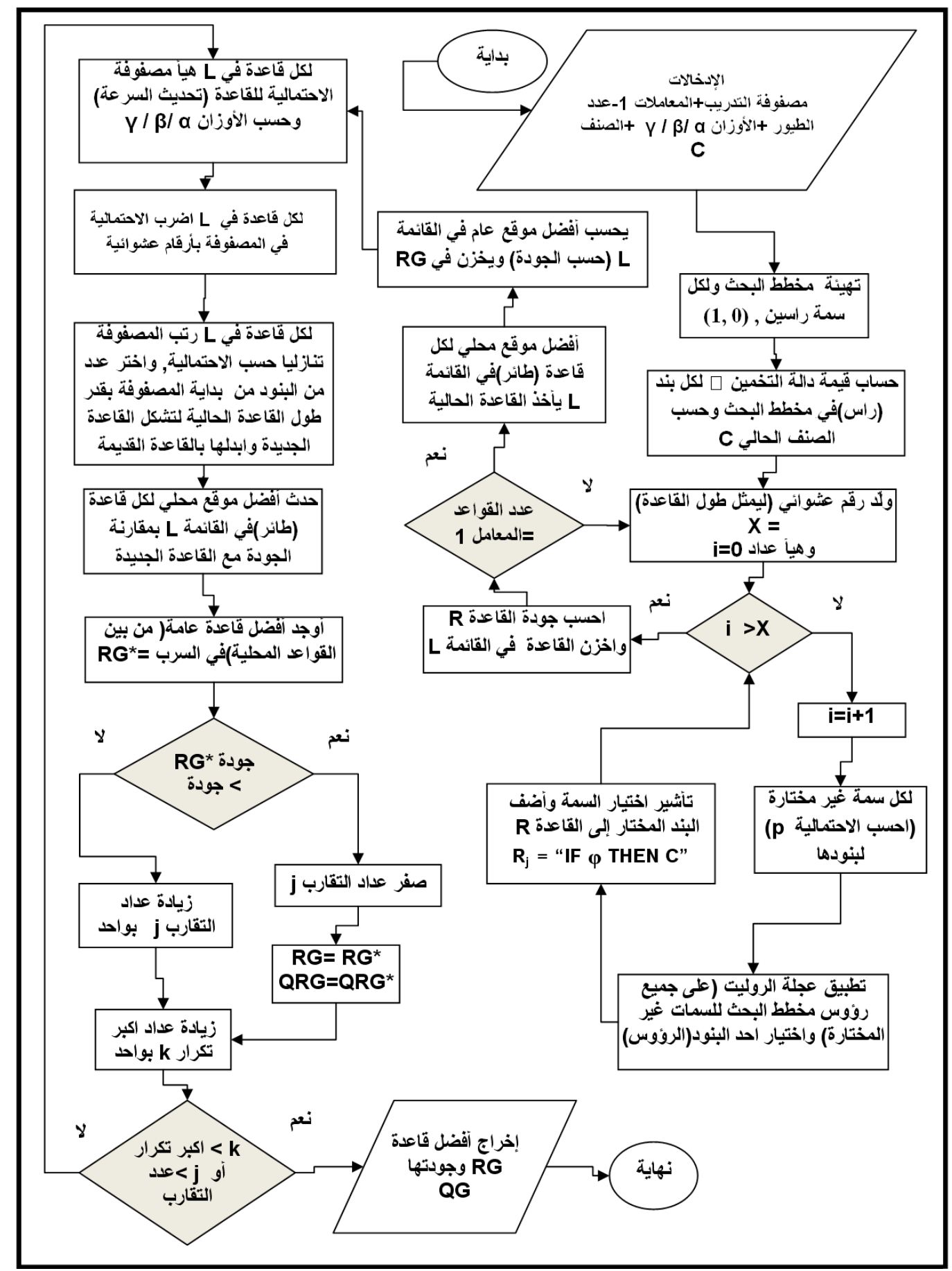

الثكل (3). المخطط الانسيابي لخوارزمية DPSO المقترحة 


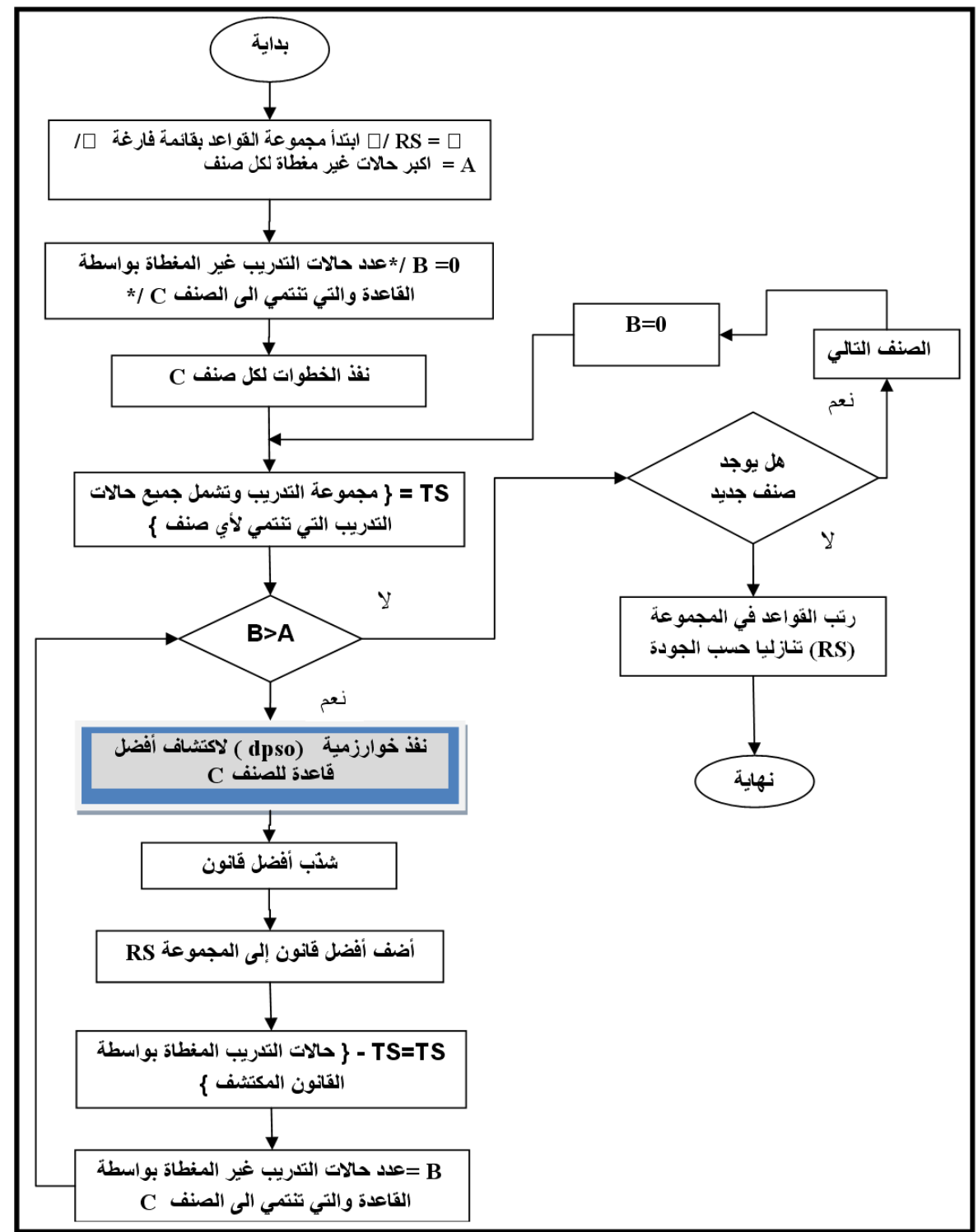

الثكل (4). المخطط الانسيابي لخوارزمية التغطية المتعاقبة

11. نتائج التطبيق العملي

وتتضمن عدّة مراحل وهي:

1-11 تجميع وتهيئة بيانات التدريب

في مجال التتقيب في الثبكة العنكبوتية، لا توجد بيانات تعد قياسية لأغراض التدريب، لذلك ولغرض تهيئة بيانات التدريب، تم تجميع (300) صفحة شبكة في ثلاثة أصناف في المجالات (الصحة، والطعام، والبيئة) من لن

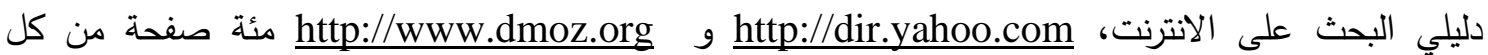
صنف، وتم تخزين تلك الصفحات في ثلاث مجلدات، وكل مجلد باسم صنف. ويقوم البرنامج بقراءة الملفات من كل مجلد وبسحب النصوص داخل الصفحات من المناطق الآتية: $<$ TITLE $>$

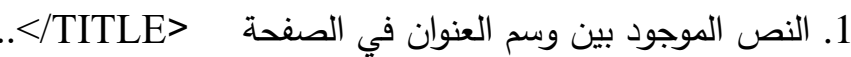


2. النص الذي يمثل الكلمات المفتاحية والموجود بعد كلمة المحتويات في الوسم الآتي:

<META NAME="keywords" CONTENT="........................">

3. النص الذي يمثل الوصف لمحتويات الصفحة والموجود بعد كلمة المحتويات في الوسم الآتي:

<"META NAME="description" CONTENT=". .">

حيث تثكل تلك المناطق الوصف الأدق لمحتوى صفحة الثبكة.

وبعد سحب النص من كل صفحة تجري العمليات التالية على النص:

1- تقطيع النص إلى كلمات (tokenized) حيث تزال جميع علامات التنقيط وتؤخذ فقط الكلمات بدون

$$
\text { فراغات وأيضاً تسمى مصطلحات (terms). }
$$

$$
\text { 3- 2- جميع الحروف تحول إلى حالة الحروف الكبيرة أو حالة الحروف الصغيرة. }
$$

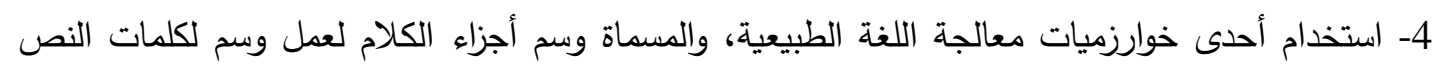
حسب تصنيف الكلمات، وبعد وسم الكلمات تسحب الأسماء فقط لأنها خير ما يعبر عن محتوى الوثائق كما في المصدر [8]، وترك باقي الكلمات.

5- حساب معامل كسب المعلومات للأسماء، وترتيبها تتازليا، واختيار عدد محدد من بداية القائمة لتمثيل

$$
\text { سمات المسألة. }
$$

وبعد ذلك يتم تثكيل مصفوفة التدريب (الوثيقة-السمة)، والتي ستمثل فضاء البحث للمسالة، الطريقة التي استخدمت في البرنامج لتمثيل مصفوفة التدريب، هي عبارة عن مصفوفة ثنائية، وأن تقاطع (الصفحة - السمة)

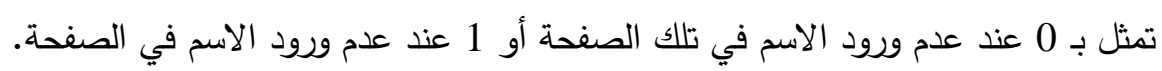

2-11 تهيئة المدخلات لخوارزمية (DPSO)

تحتاج خوارزمية (dpso) إلى قيم بعض المعاملات والأوزان التي تعد محددات في عملية توليد قواعد التصنيف وتثمل الآتي:

1. مصفوفة التدريب المولدة من مرحلة تهيئة البيانات.

2. الوزن ه والذي يمثل وزن مشاركة موقع الطائر الحالي في تحديث سرعة الطائر = 0.1.

3. الوزن م والذي يمثل وزن مشاركة أفضل موقع محلي مرّ به الطائر في تحديث سرعة الطائر =0.13.

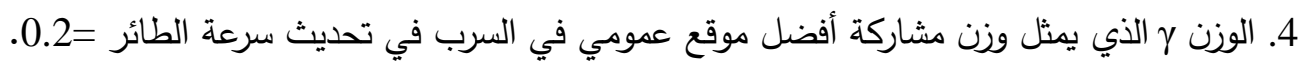

$$
\text { 5. أكبر حالات غير المغطاة لكل صنف في بيانات التدريب = } 5 .
$$

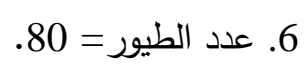

اختيرت الأوزان (

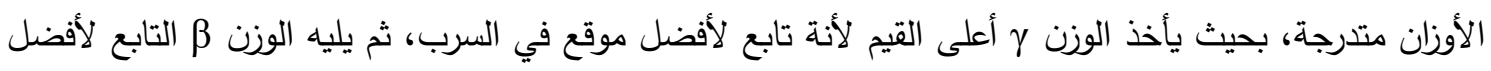

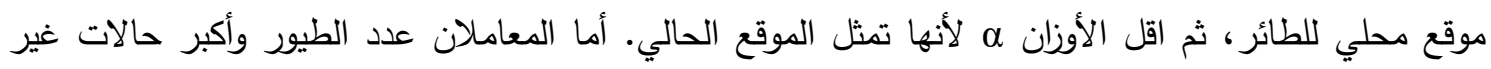

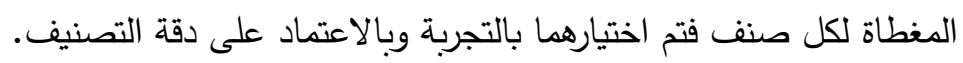




\section{Ant-miner 3-11 قياس دقة التصنيف والمقارنة مع خوارزمية}

لغرض قياس دقة التصنيف تم استخدام طريقة الطيات العشرة (ten fold cross validation)]، وفيها تقسم بيانات التدريب إلى عشرة أقسام بنسبة توزيع متساوية بين الأصناف الثلاثة وتم تتفيذ الخوارزمية بصورة

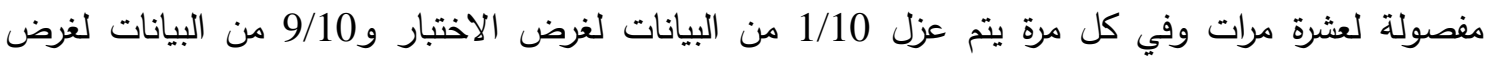

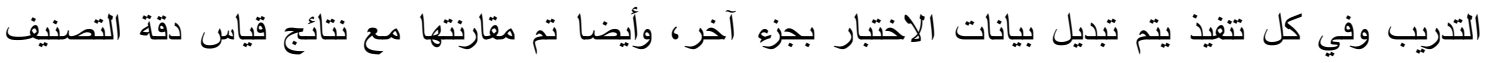
لخوارزمية النمل في التتقيب Ant-miner والمنفذّة في المصدر [2]. والجدول (4) يبين نتائج قياس دقة التصنيف للخوارزميتين وزمن التنفيذ لعشرة مرات.

جلول (4). نتائج دقة التصنيف وزمن التدريب للخوارزميتين Ant-miner و DPSO

\begin{tabular}{|c|c|c|c|c|}
\hline \multicolumn{2}{|c|}{ خوارزمية أَمثَكَة عناصر السرب } & \multicolumn{2}{|c|}{ خوارزمية النمل في التتقيب } & \multirow{2}{*}{ أجزاء التدريب والاختبار } \\
\hline زمن التدريب(ثواني) & الدقة \% & زمن التدريب(ثواني) & الدقة & \\
\hline 9.348 & $93 \pm 4.554$ & 6.640 & $93 \pm 4.554$ & 1 \\
\hline 9.542 & $93 \pm 4.279$ & 9.921 & $93 \pm 4.279$ & 2 \\
\hline 7.142 & $90 \pm 5.004$ & 13.100 & $93 \pm 4.150$ & 3 \\
\hline 9.202 & $90 \pm 5.004$ & 14.300 & $93 \pm 4.554$ & 4 \\
\hline 7.155 & $93 \pm 4.554$ & 10.450 & $96 \pm 2.984$ & 5 \\
\hline 10.531 & $87 \pm 5.681$ & 10.984 & $90 \pm 5.004$ & 6 \\
\hline 10.545 & $93 \pm 4.554$ & 14.158 & $93 \pm 4.554$ & 7 \\
\hline 10.560 & $90 \pm 5.004$ & 12.742 & $93 \pm 4.150$ & 8 \\
\hline 5.468 & $84 \pm 6.241$ & 19.840 & $84 \pm 6.241$ & 9 \\
\hline 9.938 & $77 \pm 6.928$ & 9.318 & $77 \pm 6.928$ & 10 \\
\hline 8.9431 & $89 \pm 5.150$ & 12.1453 & $90.5 \pm 5.000$ & المعدل \\
\hline
\end{tabular}

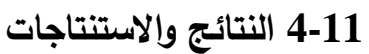

من خلال ملاحظة معدل مرات التنفيذ للخوارزميتين في الجدول (4)، فان الخوارزمية الجديدة (DPSO) تعطي دقة تصنيف 50150150 وهي نسبة مقاربة لدقة تصنيف خوارزمية النمل البالغة

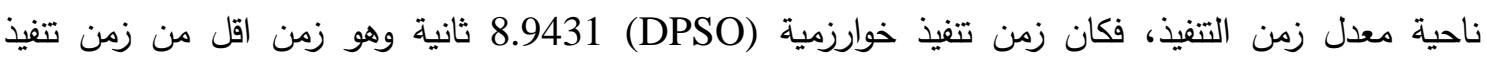

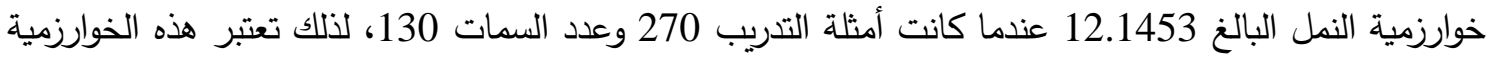
منافسة في مجال التنقيب في محتوى الثبكة العنكبوتية. 


$$
\begin{aligned}
& \text { المصادر } \\
& \text { الطويل، هالـة، (2009). "المرجـع التعليمي في التتقيب عـن البيانـات"، الطبعـة الأولى، شـعاع للنشـر } \\
& \text { والعلوم، حلب، سورية. } \\
& \text { المشهداني، محمد حامد، (2011). تقصّي التقيب في محتوى الثبكة العنكبوتية باستخدام خوارزمية أمثَّة }
\end{aligned}
$$

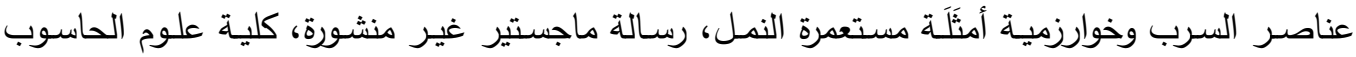

$$
\begin{aligned}
& \text { والرياضيات، جامعة الموصل. }
\end{aligned}
$$

[3] Abraham A., Grosan C. and Ramos V., (2006). "Swarm Intelligence in Data Mining", Springer-Verlag, Berlin Heidelberg.

[4] Bramer M. (2007)."Principles of Data Mining", Springer-Verlag, London.

[5] Correa E. S., Freitas A. A., and Johnson C. G., (2006). A New Discrete Particle Swarm Algorithm Applied to Attribute Selection in a Bioinformatics Data Set, Genetic and Evolutionary Computation Conference - GECCO, pp. 35-42.

[6] Correa E. S., Freitas A. A., and Johnson C. G., (2008). Particle Swarm for Attribute Selection in Bayesian Classification: An Application to Protein Function Prediction, Journal of Artificial Evolution and Applications, Vol.2008, Article ID 876746, 12 pages.

[7] Hedlund N.,(2001). Automatic Construction of Stemming Rules, Master's Thesis in Computer Science, Royal Institute of Technology, Stockholm, sweden.

[8] Holden N. and Freitas A.A., (2004). Web Page Classification with an Ant Colony Algorithm, Conference: Parallel Problem Solving from Nature - PPSN, pp.1092-1102.

[9] Holden N. P. and Freitas A. A.(2007):A Hybrid Pso/Aco Algorithm for Classification, GECCO Workshop on Particle Swarms, Second Decade, USA, pp. 2745-2750.

[10] Jurafsky D., Martin J. H. ( 2008). "Speech and Language Processing”, second edition, Prentice Hall.

[11] Liu B., (2007)."Web Data Mining Exploring Hyperlinks, Contents, and Usage Data", Springer-Verlag, Berlin Heidelberg, New York.

[12] Martens D, De Backer M, Haesen R, Snoeck M, Vanthienen J and Baesens B (2007). Classifcation with ant colony optimization, IEEE Transaction on Evolutionary Computation Vol. 11, No.5, pp.651-665.

[13] Nagel C. Evjen B. Glynn J. Skinner M. Watson K.,(2008)."Professional C\# 2008", Wiley Publishing, Inc.,Indiana.

[14] Qi X., and Davison B.D. (2009). Web Page Classifcation: Features and Algorithms, ACM Computing Surveys - CSUR , vol. 41, no. 2, pp. 1-31.

[15] Rao S.S., (2009). "Engineering Optimization Theory and Practice", Fourth Edition, JOHN WILEY \& SONS, INC., Canada. 
[16] Scime A., (2005), Web Mining Applications and Techniques, Idea Group Inc, USA.

[17] Sousa T., Silva A., and Neves A. (2004). Particle Swarm based Data Mining Algorithms for classification tasks, Parallel Computing, Vol. 30, pp.767-783.

[18] Xu G., Zhang Z., and Li L. (2011). "Web Mining and Social Networking”, Web Information Systems Engineering and Internet Technologies, Springer Science+Business Media, Australia.

[19] Yan Y., Kamath G. and Osadciw L. A., (2009). Feature Selection Optimized by Discrete Particle Swarm Optimization for Face Recognition, Proceedings of the SPIE, Volume 7306, pp. 73061W-73061W-11. 